\title{
Impact of Agricultural Experiential Learning (AEL) Module in Soil Fertility Evaluation of Malshiras Tahasil of Solapur District (India)
}

\author{
S.R. Adat", T.R. Zagade and R.G. Nalawade \\ Division of Soil Science and Agri. Chemistry, Ratnai College of Agriculture, \\ Akluj, Dist. Solapur, India \\ *Corresponding author
}

\begin{tabular}{|l|}
\hline Ke y w o r d s \\
Calcareous, \\
Organic, \\
Experiential, \\
Chemical, \\
Macronutrients. \\
\hline Article Info \\
\hline $\begin{array}{l}\text { Accepted: } \\
\text { 29 September } 2017 \\
\text { Available Online: } \\
\text { 10 October } 2017\end{array}$ \\
\hline
\end{tabular}

\section{Introduction}

The current world population is 7.5 billion as of July 2017 and it going so on. Total cultivable area in the world is about 4.9 billion ha. in 2011 which reported by Small Planet Institute. But need of food increases as todays practices which carried out are not sufficient because of that soil health is one of the most important factor for increase productivity of soil. Being important component of geosphere-biosphere system, which provides food, fiber, fodder and shelter to living matter. Soils are a vital natural resource whose proper use greatly determines the capabilities of life support system and socio-economic development of peoples. Therefore management of soil resource on scientific principles is of prime important (Sarkar et al., 2002).

For this purpose, in Agricultural experiential learning programme 2016-2017, we selected villages from Malshiras tahsils like Mandave, Phondshiras, Gursale, Dahigaon, UmbreDahigaon, Chiknevasti-Dahigaon, Pirale, Kadamwadi, Dharmpuri, Sadashivnagar (TalMalshiras) and Bhagatwadi (Tal-Indapur). 
Total 101 samples were analysed for physicochemical and chemical properties of soil. To know the present status of soil and future productivity, it is essential to know the fertility status. Considerable work on physicochemical properties of Maharastra soils was reported. (Bharambe, 2001). Thus, it is necessary to define the areas of deficiency of particular nutrients in a particular areas and crops. Soil test data would be helpful in growing such deficient areas on soil and crop basis. Thus, the details of soil resources using data on various soil properties, focus given on fertility status. Hence, present investigation is useful in judging the deficiencies of various nutrients and helpful for increasing soil fertility status.

\section{Materials and Methods}

Physico-Chemical properties in soils were determined by following methods

I. Soil pH - It was determined in 1:2.5 soil water suspension using glass electrode $\mathrm{pH}$ meter (Jackson, 1967).

II. Electrical Conductivity - Electrical conductivity was determined in 1:2.5 soil water suspension ratio after settlement of solids using conductivity bridge model and expressed in $\mathrm{dS} \mathrm{m}^{-1}$ (Jackson, 1967).

III. Organic carbon - Organic carbon of soil was estimated by wet oxidation Method which suggested by Walkely and Black. (Black 1965)

IV. Free Calcium carbonate - It was determined by rapid titration method by using $0.5 \mathrm{~N} \mathrm{HCl}$ (Black, 1965).

Available macronutrient content in soils were determined by following methods

I. Available nitrogen -Available nitrogen was determined by alkaline potassium permanganate method as suggested by Subbiah and Asija (1956).

II. Available phosphorus- The Available phosphorus was determined by Olsen's method using $0.5 \mathrm{M}$ sodium bicarbonate $(\mathrm{pH}$ 8.5) as an extracting reagent as described by (Chopra and Kanwar,1976).

III. Available potassium- The Available potassium was determined by soil treated with neutral normal ammonium acetate and the potassium in extract was determined by emission spectroscopy by flame photometer (Jackson, 1967)

\section{Results and Discussion}

\section{Status of physico-chemical properties in soils}

Soil $\mathrm{pH}$ is considered a master variable in soils as it affects many chemical processes. It specifically affects availability of nutrients by controlling the chemical forms of the different nutrients and influencing the chemical reactions they undergo. Soil electrical conductivity is an indirect measurement that correlates very well with several soil physical and chemical properties. Organic carbon might be due to addition of organic matter through either artificially or naturally and its subsequent decomposition whereas decreases with poor management and higher temperature. Calcareous soils are characterized by the presence of calcium carbonate in the parent material.and may have a calcic horizon, a layer of secondary accumulation of carbonates (usually calcium or $\mathrm{Mg}$ ) in excess of $15 \%$ calcium carbonate equivalent and at least 5\% more carbonate than an underlying layer. Hence such nutrients availability indicated in following table.

The data from total villages, $\mathrm{pH}$ range 7.00 and 8.60 with mean value of 7.86 was 
observed in Mandave (MMH3) and Chikanewasti-Dahigaon (MCD3) \& Gursale (MGD3) village respectively. Out of 101 samples, 27 per cent samples were categorized under Neutral and 73 per cent were alkaline in reaction. The relative high content of $\mathrm{pH}$ might be due to fact that these soils were developed from basaltic parent material, which are rich in basaltic cations. Gajbe et al., (1976) observed that $\mathrm{pH}$ of soils from Marathwada region ranges between 7.00 to 9.20.Similar results were reported by Aage et al., (2007) and Jibhakate et al., (2009).The electrical conductivity of these soils were ranged from 0.130 to $1.190 \mathrm{dSm}^{-1}$ with an average value of $0.544 \mathrm{dSm}^{-1}$.The lowest value of EC was recorded in village Kadamwadi (MK3), while highest EC was observed in village Sadashivnagar (MS2).

From total samples 92 percent samples were categorized under safe in EC. The low EC content of these soils might be due to leaching of salts. Padole and Mahajan (2003) revealed that EC of Swell Shrink soils of Vidharbha region were ranged from 0.13 to $1.54 \mathrm{dSm}^{-1}$. Similar findings were reported by Bharambe and Ghonsikar, (1985) and Puri et al., (2009).

The data on per cent organic carbon content revealed that the per cent organic carbon content ranges from 0.15 to 1.95 per cent with the mean value of 0.47 percent. The lowest value of organic carbon content was recorded from Gursale and Pirale (MG4 \&MPB3) village whereas, the high content of per cent organic carbon observed in ChikanewastiDahigaon (MCD1) village. Out of 101 samples 55, 35 and 10 per cent were categorized in low, medium and high content in per cent organic carbon respectively. The high content of organic carbon might be due to addition of organic matter through either artificially or naturally and its subsequent decomposition whereas, low to medium content of organic carbon in soils resemblance with poor management and higher temperature. (Waiker et al., 2004). The calcium carbonate content in these soils were ranged from 0.72 to 12.00 per cent with a mean value of 4.15 per cent. The lowest value (0.72 percent) of percent $\mathrm{CaCO}_{3}$ content was recorded in Chikanewasti-Dahigaon (MCD5) while the highest (12.00 percent) $\mathrm{CaCO}_{3}$ content observed in Dahigaon (MD4). Among 101 samples, 55 per cent were categorized in non-calcareous, 43 per cent samples were categorized in calcareous soils whereas 2 per cent samples were in highly calcareous in nature. These values indicated that the soils were non-calcareous to calcareous in nature. Puri et al., (2009) reported the status of free $\mathrm{CaCo}_{3}$ content ranged from 0.43 to 7.04 per cent. These results were in confirmity with the results of Padole and Mahajan, (2003) and Jibhakate et al., (2009).

\section{Status of Available N, P, and K in soils}

The N, P and $\mathrm{K}$ are the key nutrient, which are required for plant metabolism. Due to imbalance supply of faulty management practices there is decrease in availability of these nutrients. Hence wide spread deficiency or unavailability of nutrients in soils of tahsils undertaking for study.

The data on status of $\mathrm{N}, \mathrm{P}$ and $\mathrm{K}$ in soils of Malshiras tahsil showed in above table. The available $\mathrm{N}$ content of these soils were ranged from 40.32 to $409.5 \mathrm{Kg} \mathrm{ha}^{-1}$ with a mean value of $211.30 \mathrm{Kg} \mathrm{ha}^{-1}$. The lowest $\mathrm{N}$ content was observed in soils of Mandave(MM1) village whereas the highest $\mathrm{N}$ content were recorded in soils of Sadashivnagar (MS2). Out of 101 soil samples, 80 percent in low,19 percent medium and 1 per cent in high available $\mathrm{N}$ content. The variation in available $\mathrm{N}$ content in soils could be attributed to the differences in their physiography as well as differential cultivation and management practices. 
Table.1 Status of Physico-Chemical properties in soils of Malshiras tahsil from Solapur distrct, India

\begin{tabular}{|c|c|c|c|c|c|}
\hline Sr.No. & $\begin{array}{c}\text { Sample } \\
\text { no. }\end{array}$ & pH & $\begin{array}{c}\mathrm{EC} \\
\left(\mathbf{d S m}^{-1}\right)\end{array}$ & $\begin{array}{c}\text { Org. carbon } \\
(\%)\end{array}$ & $\begin{array}{c}\text { Free } \mathrm{CaCO}_{3} \\
(\%)\end{array}$ \\
\hline 1 & MM1 & 8.01 & 0.495 & 0.63 & 5.25 \\
\hline 2 & MM2 & 7.40 & 0.500 & 0.30 & 3.80 \\
\hline 3 & MM3 & 7.10 & 0.700 & 0.37 & 5.40 \\
\hline 4 & MM4 & 7.20 & 0.455 & 0.46 & 6.00 \\
\hline 5 & MM5 & 7.30 & 0.750 & 0.66 & 5.50 \\
\hline 6 & MP1 & 7.30 & 0.760 & 0.58 & 1.87 \\
\hline 7 & MP2 & 7.90 & 0.282 & 0.39 & 3.12 \\
\hline 8 & MP3 & 7.50 & 0.470 & 0.48 & 2.50 \\
\hline 9 & MP4 & 7.60 & 0.510 & 0.55 & 2.00 \\
\hline 10 & MP5 & 7.40 & 0.370 & 0.72 & 5.00 \\
\hline 11 & MG1 & 7.33 & 0.735 & 0.27 & 7.76 \\
\hline 12 & MG2 & 7.40 & 0.730 & 0.28 & 7.70 \\
\hline 13 & MG3 & 7.79 & 0.650 & 0.49 & 5.75 \\
\hline 14 & MG4 & 7.20 & 0.690 & 0.15 & 1.87 \\
\hline 15 & MG5 & 7.90 & 0.940 & 0.75 & 2.10 \\
\hline 16 & MG6 & 7.30 & 0.590 & 0.27 & 7.67 \\
\hline 17 & MG7 & 7.40 & 0.680 & 0.85 & 8.50 \\
\hline 18 & MG8 & 7.40 & 0.730 & 0.27 & 7.76 \\
\hline 19 & MG9 & 7.78 & 0.650 & 0.49 & 5.75 \\
\hline 20 & MD1 & 7.50 & 0.470 & 0.48 & 2.50 \\
\hline 21 & MD2 & 7.40 & 0.370 & 0.72 & 5.00 \\
\hline 22 & MD3 & 7.38 & 0.760 & 0.58 & 1.87 \\
\hline 23 & MD4 & 7.65 & 0.570 & 0.55 & 12.0 \\
\hline 24 & MD5 & 7.95 & 0.280 & 0.39 & 3.12 \\
\hline 25 & MUD1 & 7.90 & 0.510 & 0.37 & 4.50 \\
\hline 26 & MUD2 & 7.60 & 0.550 & 0.55 & 7.20 \\
\hline 27 & MUD3 & 7.60 & 0.870 & 0.65 & 8.25 \\
\hline 28 & MUD4 & 7.90 & 0.280 & 0.39 & 3.12 \\
\hline
\end{tabular}


Int.J.Curr.Microbiol.App.Sci (2017) 6(10): 5005-5017

\begin{tabular}{|c|c|c|c|c|c|}
\hline 29 & MUD5 & 7.75 & 0.400 & 0.19 & 4.25 \\
\hline 30 & MCD1 & 7.40 & 0.850 & 1.95 & 2.00 \\
\hline 31 & MCD2 & 7.80 & 0.800 & 1.20 & 1.52 \\
\hline 32 & MCD3 & 8.60 & 0.860 & 0.38 & 6.21 \\
\hline 33 & MCD4 & 8.50 & 0.550 & 0.81 & 12.15 \\
\hline 34 & MCD5 & 7.50 & 0.480 & 0.60 & 0.72 \\
\hline 35 & MDD1 & 7.60 & 0.490 & 0.19 & 2.50 \\
\hline 36 & MDD2 & 7.30 & 0.350 & 0.28 & 2.50 \\
\hline 37 & MDD3 & 8.30 & 0.440 & 0.25 & 1.25 \\
\hline 38 & MDD4 & 7.79 & 0.650 & 0.18 & 5.75 \\
\hline 39 & MDD5 & 8.20 & 0.420 & 0.30 & 1.50 \\
\hline 40 & MGD1 & 8.30 & 0.750 & 0.56 & 5.00 \\
\hline 41 & MGD2 & 8.50 & 0.760 & 0.52 & 4.00 \\
\hline 42 & MGD3 & 8.60 & 0.780 & 0.51 & 4.00 \\
\hline 43 & MGD4 & 8.40 & 0.740 & 0.52 & 5.00 \\
\hline 44 & MGD5 & 8.30 & 0.150 & 0.53 & 3.00 \\
\hline 45 & MB1 & 8.50 & 0.740 & 0.52 & 5.00 \\
\hline 46 & MB2 & 8.20 & 0.520 & 0.45 & 3.50 \\
\hline 47 & MB3 & 8.40 & 0.130 & 0.54 & 2.00 \\
\hline 48 & MB4 & 8.00 & 0.230 & 0.37 & 2.50 \\
\hline 49 & MB5 & 8.50 & 0.380 & 0.22 & 3.00 \\
\hline 50 & MPP1 & 8.40 & 0.740 & 0.52 & 5.20 \\
\hline 51 & MPP2 & 8.30 & 0.140 & 0.53 & 2.00 \\
\hline 52 & MPP3 & 8.00 & 0.230 & 0.36 & 2.50 \\
\hline 53 & MPP4 & 8.20 & 0.520 & 0.42 & 3.50 \\
\hline 54 & MPP5 & 8.50 & 0.380 & 0.22 & 3.00 \\
\hline 55 & MUDH1 & 7.70 & 0.730 & 0.25 & 4.25 \\
\hline 56 & MUDH2 & 8.50 & 0.740 & 0.20 & 4.20 \\
\hline 57 & MUDH3 & 8.40 & 0.130 & 0.54 & 2.00 \\
\hline 58 & MUDH4 & 8.00 & 0.230 & 0.37 & 2.50 \\
\hline 59 & MUDH5 & 8.60 & 0.380 & 0.22 & 3.00 \\
\hline 60 & MPB1 & 7.60 & 0.730 & 0.25 & 4.25 \\
\hline
\end{tabular}


Int.J.Curr.Microbiol.App.Sci (2017) 6(10): 5005-5017

\begin{tabular}{|c|c|c|c|c|c|}
\hline 61 & MPB2 & 7.90 & 0.720 & 0.20 & 4.20 \\
\hline 62 & MPB3 & 7.50 & 0.750 & 0.15 & 4.50 \\
\hline 63 & MPB4 & 7.20 & 0.770 & 0.25 & 4.80 \\
\hline 64 & MPB5 & 7.40 & 0.750 & 0.50 & 4.50 \\
\hline 65 & MK1 & 8.50 & 0.240 & 0.50 & 5.00 \\
\hline 66 & MK2 & 8.20 & 0.520 & 0.45 & 3.50 \\
\hline 67 & MK3 & 8.40 & 0.130 & 0.54 & 2.00 \\
\hline 68 & MK4 & 8.00 & 0.300 & 0.37 & 2.50 \\
\hline 69 & MK5 & 8.50 & 0.380 & 0.22 & 3.00 \\
\hline 70 & MK6 & 8.40 & 0.740 & 0.52 & 5.20 \\
\hline 71 & MK7 & 8.20 & 0.520 & 0.42 & 3.50 \\
\hline 72 & MK8 & 8.30 & 0.140 & 0.53 & 2.00 \\
\hline 73 & MK9 & 8.00 & 0.230 & 0.36 & 2.50 \\
\hline 74 & MK10 & 8.50 & 0.380 & 0.20 & 3.00 \\
\hline 75 & MD1 & 8.20 & 0.520 & 0.45 & 3.50 \\
\hline 76 & MD2 & 8.50 & 0.740 & 0.52 & 5.00 \\
\hline 77 & MD3 & 8.40 & 0.130 & 0.54 & 2.00 \\
\hline 78 & MD4 & 8.00 & 0.230 & 0.37 & 2.50 \\
\hline 79 & MD5 & 8.50 & 0.380 & 0.22 & 3.00 \\
\hline 80 & MPY1 & 8.54 & 0.740 & 0.52 & 5.00 \\
\hline 81 & MPY2 & 8.58 & 0.380 & 0.22 & 3.00 \\
\hline 82 & MPY3 & 8.25 & 0.520 & 0.45 & 3.50 \\
\hline 83 & MPY4 & 8.58 & 0.740 & 0.52 & 5.00 \\
\hline 84 & MPY5 & 8.00 & 0.230 & 0.37 & 2.50 \\
\hline 85 & MS1 & 7.70 & 1.080 & 0.19 & 0.75 \\
\hline 86 & MS2 & 8.30 & 1.190 & 0.76 & 2.00 \\
\hline 87 & MS3 & 7.20 & 0.450 & 0.97 & 3.20 \\
\hline 88 & MS4 & 7.68 & 0.460 & 0.79 & 4.50 \\
\hline 89 & MS5 & 7.24 & 0.860 & 1.13 & 8.50 \\
\hline 90 & MMH1 & 8.02 & 0.490 & 0.53 & 6.00 \\
\hline 91 & ММH2 & 7.20 & 0.400 & 0.20 & 4.00 \\
\hline 92 & MMH3 & 7.00 & 0.600 & 0.30 & 4.00 \\
\hline
\end{tabular}


Int.J.Curr.Microbiol.App.Sci (2017) 6(10): 5005-5017

\begin{tabular}{|c|c|c|c|c|c|}
93 & MMH4 & 7.40 & $\mathbf{0 . 5 0 0}$ & $\mathbf{0 . 4 0}$ & $\mathbf{6 . 0 0}$ \\
\hline 94 & MMH5 & $\mathbf{7 . 2 0}$ & $\mathbf{0 . 6 0 0}$ & $\mathbf{0 . 8 8}$ & $\mathbf{6 . 0 0}$ \\
\hline 95 & MSS1 & $\mathbf{7 . 3 0}$ & $\mathbf{0 . 7 6 0}$ & $\mathbf{0 . 5 2}$ & $\mathbf{2 . 7 5}$ \\
\hline 96 & MSS2 & $\mathbf{7 . 5 0}$ & $\mathbf{0 . 4 7 0}$ & $\mathbf{0 . 9 8}$ & $\mathbf{8 . 2 5}$ \\
\hline 97 & MSS3 & $\mathbf{7 . 9 0}$ & $\mathbf{0 . 3 7 0}$ & $\mathbf{0 . 7 2}$ & $\mathbf{5 . 0 0}$ \\
\hline 98 & MSS4 & $\mathbf{7 . 4 5}$ & $\mathbf{0 . 8 8 0}$ & $\mathbf{0 . 6 3}$ & $\mathbf{4 . 2 5}$ \\
\hline 99 & MSS5 & $\mathbf{7 . 6 0}$ & $\mathbf{0 . 8 4 0}$ & $\mathbf{0 . 3 0}$ & $\mathbf{5 . 0 0}$ \\
\hline 100 & MSS6 & $\mathbf{7 . 9 0}$ & $\mathbf{0 . 3 7 0}$ & $\mathbf{0 . 7 2}$ & $\mathbf{5 . 0 0}$ \\
\hline 101 & MSS7 & $\mathbf{7 . 3 0}$ & $\mathbf{0 . 7 6 0}$ & $\mathbf{0 . 3 2}$ & $\mathbf{2 . 7 5}$ \\
\hline MEAN & & $\mathbf{7 . 8 6}$ & $\mathbf{0 . 5 4 4}$ & $\mathbf{0 . 4 7}$ & $\mathbf{4 . 1 5}$ \\
\hline
\end{tabular}

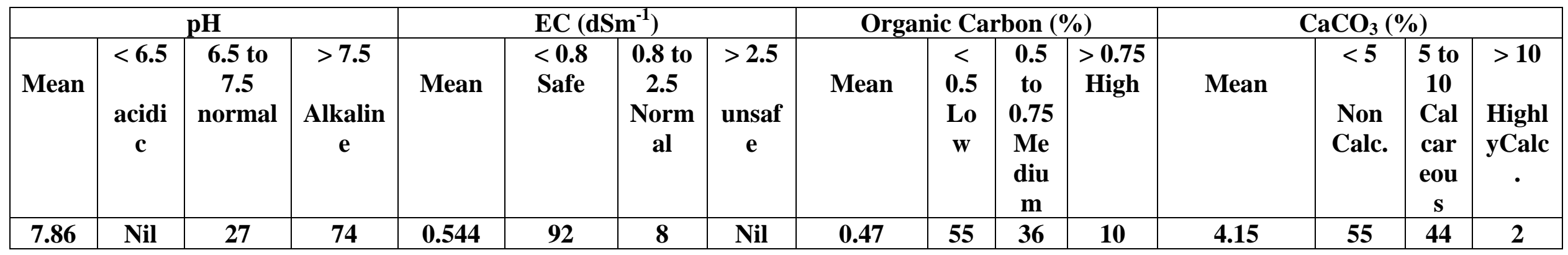


Table.2 Status of available N, P and K in soils of Malshiras tahsil from Solapur distrct, India

\begin{tabular}{|c|c|c|c|c|}
\hline $\begin{array}{l}\text { Sr. } \\
\text { No. }\end{array}$ & $\begin{array}{c}\text { Sample } \\
\text { No. }\end{array}$ & $\begin{array}{c}\mathrm{N} \\
\left(\mathrm{Kg} \mathrm{ha}^{-1}\right)\end{array}$ & $\begin{array}{c}P \\
\left(\mathrm{Kg} \mathrm{ha}^{-1}\right)\end{array}$ & $\begin{array}{c}\mathrm{K} \\
\left(\mathrm{Kg} \mathrm{ha}^{-1}\right)\end{array}$ \\
\hline 1 & MM1 & 40.32 & 10.20 & 454.2 \\
\hline 2 & MM2 & 175 & 12.20 & 345 \\
\hline 3 & MM3 & 162 & 14.10 & 377 \\
\hline 4 & MM4 & 174.4 & 8.77 & 317 \\
\hline 5 & MM5 & 189 & 16.00 & 347 \\
\hline 6 & MP1 & 409.5 & 21.68 & 308 \\
\hline 7 & MP2 & 279 & 13.77 & 280 \\
\hline 8 & MP3 & 341 & 19.13 & 327 \\
\hline 9 & MP4 & 220 & 5.00 & 281 \\
\hline 10 & MP5 & 270 & 7.14 & 716 \\
\hline 11 & MG1 & 180 & 8.60 & 380 \\
\hline 12 & MG2 & 189 & 9.60 & 382 \\
\hline 13 & MG3 & 222 & 7.60 & 572 \\
\hline 14 & MG4 & 280 & 9.18 & 425 \\
\hline 15 & MG5 & 339 & 9.40 & 414 \\
\hline 16 & MG6 & 189 & 8.81 & 403 \\
\hline 17 & MG7 & 122.5 & 7.65 & 582 \\
\hline 18 & MG8 & 189 & 8.67 & 380 \\
\hline 19 & MG9 & 222 & 7.60 & 572 \\
\hline 20 & MD1 & 341 & 19.13 & 327 \\
\hline 21 & MD2 & 270 & 7.14 & 716 \\
\hline 22 & MD3 & 409 & 21.68 & 308 \\
\hline 23 & MD4 & 220 & 5.00 & 281 \\
\hline 24 & MD5 & 273 & 13.77 & 280 \\
\hline
\end{tabular}


Int.J.Curr.Microbiol.App.Sci (2017) 6(10): 5005-5017

\begin{tabular}{|c|c|c|c|c|}
\hline 25 & MUD1 & 124 & 10.20 & 448 \\
\hline 26 & MUD2 & 220 & 5.00 & 281 \\
\hline 27 & MUD3 & 112 & 20.6 & 403.2 \\
\hline 28 & MUD4 & 273 & 13.77 & 280 \\
\hline 29 & MUD5 & 118 & 10.29 & 425 \\
\hline 30 & MCD1 & 145 & 10.20 & 362 \\
\hline 31 & MCD2 & 281 & 8.12 & 145 \\
\hline 32 & MCD3 & 172 & 11.40 & 372 \\
\hline 33 & MCD4 & 200 & 25.02 & 302.21 \\
\hline 34 & MCD5 & 290 & 14.18 & 180 \\
\hline 35 & MDD1 & 117 & 10.21 & 246 \\
\hline 36 & MDD2 & 135 & 5.90 & 243 \\
\hline 37 & MDD3 & 199 & 21.68 & 301.6 \\
\hline 38 & MDD4 & 153 & 7.60 & 572.18 \\
\hline 39 & MDD5 & 142 & 20.68 & 363.2 \\
\hline 40 & MGD1 & 240 & 14.20 & 259 \\
\hline 41 & MGD2 & 230 & 16 & 255 \\
\hline 42 & MGD3 & 220 & 18 & 260 \\
\hline 43 & MGD4 & 210 & 17.60 & 256 \\
\hline 44 & MGD5 & 200 & 33 & 240 \\
\hline 45 & MB1 & 210 & 17.40 & 256 \\
\hline 46 & MB2 & 202 & 23 & 240 \\
\hline 47 & MB3 & 212 & 32 & 235 \\
\hline 48 & MB4 & 190 & 15 & 260 \\
\hline 49 & MB5 & 185 & 4.50 & 280 \\
\hline 50 & MPP1 & 220 & 17.5 & 256.50 \\
\hline 51 & MPP2 & 220 & 3.20 & 230 \\
\hline 52 & MPP3 & 190 & 14 & 260 \\
\hline
\end{tabular}


Int.J.Curr.Microbiol.App.Sci (2017) 6(10): 5005-5017

\begin{tabular}{|c|c|c|c|c|}
\hline 53 & MPP4 & 210 & 23 & 240 \\
\hline 54 & MPP5 & 185 & 4.50 & 280 \\
\hline 55 & MUDH1 & 210 & 10.20 & 284 \\
\hline 56 & MUDH2 & 200 & 10.14 & 300 \\
\hline 57 & MUDH3 & 212 & 3.20 & 235 \\
\hline 58 & MUDH4 & 190 & 15 & 200 \\
\hline 59 & MUDH5 & 185 & 4.50 & 280 \\
\hline 60 & MPB1 & 220 & 10.20 & 284 \\
\hline 61 & MPB2 & 200 & 10.14 & 300 \\
\hline 62 & MPB3 & 350 & 15 & 150 \\
\hline 63 & MPB4 & 121 & 10 & 100 \\
\hline 64 & MPB5 & 280 & 13 & 150 \\
\hline 65 & MK1 & 210 & 17.40 & 256 \\
\hline 66 & MK2 & 202 & 2.30 & 240 \\
\hline 67 & MK3 & 212 & 3.20 & 335 \\
\hline 68 & MK4 & 190 & 15 & 260 \\
\hline 69 & MK5 & 135 & 4.50 & 280 \\
\hline 70 & MK6 & 220 & 17.5 & 256 \\
\hline 71 & MK7 & 210 & 2.30 & 240 \\
\hline 72 & MK8 & 220 & 3.20 & 230 \\
\hline 73 & MK9 & 100 & 14 & 260 \\
\hline 74 & MK10 & 185 & 4.50 & 280 \\
\hline 75 & MD1 & 202 & 2.30 & 240 \\
\hline 76 & MD2 & 210 & 1.74 & 256 \\
\hline 77 & MD3 & 212 & 3.20 & 235 \\
\hline 78 & MD4 & 190 & 11.5 & 260 \\
\hline 79 & MD5 & 185 & 4.50 & 280 \\
\hline 80 & MPY1 & 210 & 1.74 & 256 \\
\hline
\end{tabular}


Int.J.Curr.Microbiol.App.Sci (2017) 6(10): 5005-5017

\begin{tabular}{|c|c|c|c|c|c|c|c|c|c|c|c|}
\hline & & & 81 & MPY2 & \multicolumn{2}{|c|}{185} & 4.50 & \multicolumn{2}{|l|}{280} & & \\
\hline & & & 82 & MPY3 & \multicolumn{2}{|c|}{202} & 2.30 & \multicolumn{2}{|l|}{240} & & \\
\hline & & & 83 & MPY4 & \multicolumn{2}{|c|}{210} & 1.74 & \multicolumn{2}{|l|}{236} & & \\
\hline & & & 84 & MPY5 & \multicolumn{2}{|c|}{190} & 15 & \multicolumn{2}{|l|}{260} & & \\
\hline & & & 85 & MS1 & \multicolumn{2}{|c|}{136} & 3.75 & \multicolumn{2}{|l|}{381} & & \\
\hline & & & 86 & MS2 & \multicolumn{2}{|c|}{532} & 22.89 & \multicolumn{2}{|l|}{223} & & \\
\hline & & & 87 & MS3 & \multicolumn{2}{|c|}{341} & 23 & \multicolumn{2}{|l|}{627} & & \\
\hline & & & 88 & MS4 & \multicolumn{2}{|c|}{296} & 15.18 & \multicolumn{2}{|l|}{582.8} & & \\
\hline & & & 89 & MS5 & \multicolumn{2}{|c|}{327.7} & 24.49 & \multicolumn{2}{|l|}{352.4} & & \\
\hline & & & 90 & MMH1 & \multicolumn{2}{|c|}{45} & 8.00 & \multicolumn{2}{|l|}{275} & & \\
\hline & & & 91 & MMH2 & \multicolumn{2}{|c|}{172} & 12.70 & \multicolumn{2}{|l|}{310} & & \\
\hline & & & 92 & ММH3 & \multicolumn{2}{|c|}{170} & 18 & \multicolumn{2}{|l|}{340} & & \\
\hline & & & 93 & MMH4 & \multicolumn{2}{|c|}{175} & 11.77 & \multicolumn{2}{|l|}{317} & & \\
\hline & & & 94 & MMH5 & \multicolumn{2}{|c|}{190} & 10 & \multicolumn{2}{|l|}{290} & & \\
\hline & & & 95 & MSS1 & \multicolumn{2}{|c|}{208} & 8.67 & \multicolumn{2}{|l|}{375} & & \\
\hline & & & 96 & MSS2 & & & 8.26 & 515 & & & \\
\hline & & & 97 & MSS3 & & & 7.40 & 480 & & & \\
\hline & & & 98 & MSS4 & & & 8.76 & 380 & & & \\
\hline & & & 99 & MSS5 & & & 8.90 & 444 & & & \\
\hline & & & 100 & MSS6 & & & 7.14 & 716 & & & \\
\hline & & & 101 & MSS7 & & & 8.76 & 403 & & & \\
\hline & & & MEAN & & & & 11.49 & 326.53 & & & \\
\hline & & $\left.\mathrm{g} \mathrm{ha}^{-1}\right)$ & & & & $\left.h^{-1}\right)$ & & & & $\left(\mathrm{kg} \mathrm{ha}^{-1}\right)$ & \\
\hline Mean & $\begin{array}{l}<250 \\
\text { Low }\end{array}$ & $\begin{array}{c}250 \text { to } \\
500 \\
\text { Medium }\end{array}$ & $\begin{array}{l}>500 \\
\text { High }\end{array}$ & Mean & $\begin{array}{l}<10 \\
\text { Low }\end{array}$ & $\begin{array}{c}10 \text { to } 25 \\
\begin{array}{c}\text { Mediu } \\
\text { m }\end{array}\end{array}$ & $\begin{array}{l}>25 \\
\text { High }\end{array}$ & Mean & $\begin{array}{l}<150 \\
\text { Low }\end{array}$ & $\begin{array}{l}150 \text { to } 300 \\
\text { Medium }\end{array}$ & $\begin{array}{l}>300 \\
\text { High }\end{array}$ \\
\hline 211.30 & 81 & 19 & 1 & 11.49 & 48 & 50 & 3 & 326.53 & 2 & 53 & 46 \\
\hline
\end{tabular}


Table.3 Nutrient Index values for the soil samples

\begin{tabular}{|c|c|c|}
\hline Characteristics & Nutrient Index Value & Remarks \\
\hline Nitrogen & 1.21 & Low \\
\hline Phosphorus & 1.53 & Medium \\
\hline Potassium & 2.44 & Medium \\
\hline
\end{tabular}

Patil et al., (1994) reported that losses due to leaching and denitrification resulted in low amount of available $\mathrm{N}$ in soil. The available phosphorus content in these soils were varied from lowest $\mathrm{P}\left(1.74 \mathrm{Kg} \mathrm{ha}^{-1}\right)$ in Dharmpuri (MD2), Phondshiras (MPY1,MPY4) to $33 \mathrm{Kg}$ $\mathrm{ha}^{-1}$ with a mean value of $11.49 \mathrm{Kg} \mathrm{ha}^{-1}$. These results clearly showed that 48 per cent low, 50 per cent medium and 2 per cent high that is soils of Malshiras tahsil were low to medium in $\mathrm{P}$ content. The large range may be due to variation in soil properties and their high $\mathrm{P}$ fixing capacity, which prevents to come into readily available form in soil solution. These results were confirmity with Adat (2010) and Puri, (2009). The large range may be due to variation in soil properties and their high $\mathrm{P}$ fixing capacity, which prevents to come into readily available form in soil solution.

The available potassium contents in these soils were ranged from 100 to $716 \mathrm{Kg} \mathrm{ha}^{-1}$ with an average value of $326.53 \mathrm{Kg} \mathrm{ha}^{-1} \mathrm{~K}$. The lowest value of $\mathrm{K}$ was recorded in soils of Pirale (MPB4) while highest value of K content was recorded in Phondshiras (MP5) and Dahigaon (MD2) village respectively. Out of 101 samples 2, 52 and 46 percent samples were categorized in low, medium and high in $\mathrm{K}$ content respectively. The value indicated that the soils of Malshiras tahsil were medium to high $\mathrm{K}$ content. These results were in confirmity with results of Patil and Sonar, (1994) reported that the available K content in soils of Maharashtra was varied from 224 to $909 \mathrm{Kg} \mathrm{ha}^{-1}$. Similar results were reporte by Waghmare, (2009)

\section{Soil Nutrient Index of soils of Malshiras} tahsil

Considering "Soil Nutrient Index" soils of Malshiras tahsil were found in category 'Low' fertility status for nitrogen, 'Medium' for phosphorous and 'Medium' with respect to potassium. The values worked out from nutrient index for nitrogen, Phosphorous and Potassium were 1.21, 1.53 and 2.44 respectively, against the nutrient index values $<1.50$ for low, 1.50 to 2.50 for medium and $>$ 2.50 for high fertility status.

The present investigation was carried by students of Agricultural Experiential Learning Module to study chemical properties and available nutrient status from the soils of Malshiras tahsil of Solapur district. The soils were neutral to alkaline in reaction with average $\mathrm{pH}$ 7.86, The soils were categorized as safe in respect of electrical conductivity with mean of $0.544 \mathrm{dS} / \mathrm{m}$ which safe in soluble salt concentration, O.C- $0.47 \%$ which shows low to medium and $\mathrm{CaCO}_{3} 4.15 \%$ which indicates calcareous condition.In general, it showed that the properties like $\mathrm{pH}$, $\mathrm{EC}$, Organic carbon and $\mathrm{CaCO}_{3}$ contents were found to be decide the availability of nutrients in these soils. In chemical properties, macronutrients from the result, average available Nitrogen present about 211.30 $\mathrm{Kg} / \mathrm{ha}$ which shows low availability of Nitrogen, available Phosphorus $11.49 \mathrm{Kg} / \mathrm{ha}$ and available Potassium $326.53 \mathrm{Kg} / \mathrm{ha}$ which indicates low to medium status of $\mathrm{P}$ but Potassium content is medium to high in Malshiras tahsil soils. Thus according to soil nutrient index, it can be concluded that soils 
of Malshiras tahsil are low to medium in fertility status.

\section{References}

Aage, A.B., Magar S.M., Godhawale G.V. and Borgaonkar S.B. 2007b. Studies on available macronutrients status in Beed district of Maharashtra state. Int. J. of Tropical Agriculture, 25 (3): 487-489.

Adat, S.R., 2010. Soil fertility mapping of Hingoli and Sengaon tahsils of Hingoli district. M.Sc. (Agri.) thesis submitted to M.A.U. Parbhani.

Bharambe P.R., and Ghonshikar C.P. 1985. Physico-chemical charaeteristics of soils in Jayakwadi command. $J$. Maharashtra agric. Univ., 10 (3): 247249.

Bharmbe P.R., and Ghonshikar C.P. 1984. Fertility status of soils in Jayakwadi command. J.maharastra agric. Univ., 9(3): 326-327.

Chopra, S.L., and Kanwar, J.B. 1976. Analytical Agricultural Chemistry. Kalyani publ., New Delhi.

Gajbe, M.V., Londe, M.G. and Varade, S.B. 1976. Soils of Marathwada. J. Maharashtra Agric. Univ. 1 (2-6): 5559.

Jackson, M.L., 1967. Soil chemical analysis, Prentice Hall of India pvt. Ltd, New Delhi. 46,128-135 and 283.

Jibhakate, S.B., Bhende, S.M., Kharche V.K. and Selvalakshmi V. 2009. Physicochemical status of soils of Katol tahsil in Nagpur district J.Soils and crops 19 (1): 122-128.

Padole, V.R., and Mahajan, S.B. 2003. Status and release of Potassium in some swellshrink soils of Vidharbha, Maharashtra. J. Maharashtra Agric. Univ. 28 (1): 3-7.

Patil, Y.M., and Sonar, K.R. 1994. Status of major and micronutrient of swell-shrink soils of Maharashtra. J. Maharashtra agric. Univ. 19 (2):169.

Puri, A.N., Khandekar, B.S., Suryavanshi, V.P and Pagar, P.A. 2009. Assessment of molybdenum and Boron status of Junner and Haveli tahsil of Pune district of Maharashtra. IJTA serials publication 27 (3-4): 521-524.

Subhiah, B.V., and Asija, G.L. 1956. Rapid procedure for the estimation of available nitrogen in soil. Curr.Sci.J.25: 259-260.

Waghmare, M.S., 2007. Studies on Nutrient Availability of soils of Ausa and Nilanga tahsils of Latur district. M.sc (Agri) thesis submitted to MAU parbhani.

Waikar, S.L., Malewar, G.V. and More, S.D. 2004. Elemental composition of humic and fluvic acids in the soils of Marathwada region of Maharashtra. $J$. Maharashtra agric. Univ. 21 (2): 127129.

\section{How to cite this article:}

Adat, S.R., T.R. Zagade and Nalawade, R.G. 2017. Impact of Agricultural Experiential Learning (AEL) Module in Soil Fertility Evaluation of Malshiras Tahasil of Solapur District (India). Int.J.Curr.Microbiol.App.Sci. 6(10): 5005-5017. doi: https://doi.org/10.20546/ijcmas.2017.610.474 